\title{
Lidil
}

Revue de linguistique et de didactique des langues

47 | 2013

Le verbe pour exprimer le temps

\section{De l'analyse de productions écrites d'élèves et de ses usages potentiels pour la formation des enseignants du secondaire en grammaire}

Roxane Gagnon

\section{OpenEdition}

\section{Journals}

Édition électronique

URL : http://journals.openedition.org/lidil/3261

DOI : 10.4000/lidil.3261

ISSN : 1960-6052

Éditeur

UGA Éditions/Université Grenoble Alpes

\section{Édition imprimée}

Date de publication : 31 mai 2013

Pagination : 21-40

ISBN : 978-2-84310-247-9

ISSN : $1146-6480$

Référence électronique

Roxane Gagnon, « De l'analyse de productions écrites d'élèves et de ses usages potentiels pour la formation des enseignants du secondaire en grammaire », Lidil [En ligne], 47 | 2013, mis en ligne le 01 décembre 2014, consulté le 01 mai 2019. URL : http://journals.openedition.org/lidil/3261 ; DOI : 10.4000/lidil.3261 


\title{
De l'analyse de productions écrites d'élèves et de ses usages potentiels pour la formation des enseignants du secondaire en grammaire
}

\author{
Roxane Gagnon*
}

\begin{abstract}
RÉSUMÉ
Cet article présente une proposition de traitement didactique du fonctionnement des temps du verbe qui prend appui sur l'analyse de textes d'élèves. La démarche d'analyse examine les composantes génériques du texte, la mise en forme discursive ainsi que les dimensions liées à la grammaire de la phrase. Des analyses de textes argumentatifs de corpus contrastés viennent illustrer la démarche adoptée. Enfin, les usages potentiels de cette démarche d'analyse dans le contexte de la formation des enseignants du secondaire sont présentés.
\end{abstract}

\section{ABSTRACT}

This paper develops and proposes a didactic treatment of verb tense, based on the analysis of texts produced by students. It examines the generic components of the text, its discursive layout, and dimensions in relation to sentence grammar. The study is illustrated with a comparative analysis of argumentative texts. Finally, this paper proposes potential uses of this analytical approach in the training of secondary school teachers.

«Anyone writing a book on time in language has to face two problems which are also found elsewhere, but hardly to the same extent. The first is the amount of research on the subject [...] The second problem is what one might call

* GRAFE, Université de Genève.

L'acronyme GRAFE signifie Groupe de recherche pour l'analyse du français enseigné. Il regroupe principalement des chercheurs de la Faculté de psychologie et des sciences de l'éducation de l'université de Genève. 


\begin{abstract}
the "ECE-problem of research": everything is connected to everything. Temporality involves lexical semantics, deixis and context-dependency of meaning, inflectional morphology, problems of quantification, word order and other aspects of syntax-it is connected to everything. But somewhere there must be a limit to the discussion, and the line is often not easily drawn.»
\end{abstract}

W. KLEIN (1994),

Time in Language, p. XII.

S'il est un problème persistant de l'enseignement de la grammaire, c'est sans aucun doute le décalage existant entre prescriptions officielles et pratiques effectives des enseignants (Schneuwly, Dolz et coll., 2009). Ce décalage est visible dans les diverses terminologies en usage dans les plans d'étude, les moyens d'enseignement et le discours des enseignants (Chartrand \& de Pietro, 2012). En outre, les obstacles observés dans les pratiques langagières des élèves sont souvent bien éloignés des contenus proposés par les plans d'études (Marmy Cusin, 2012). Comme ils peinent à articuler et à intégrer les activités grammaticales à l'intérieur d'activités de communication, les enseignants préfèrent faire de la grammaire de façon détachée, hors contexte.

Il n'en demeure pas moins que l'étude de certains phénomènes grammaticaux demande de s'éloigner d'une grammaire de la phrase pour passer par le texte et l'activité langagière dans sa globalité. C'est le cas de l'emploi des temps verbaux. Or, l'enseignement des valeurs et du fonctionnement des formes verbales souffre depuis longtemps d'un problème de contexte : la plupart des manuels et grammaires scolaires ramènent la question à la présentation de quelques exemples soigneusement choisis, limitant au cadre de la phrase une réalité complexe inscrite dans le texte (Combettes \& Fresson, 1975). Et ce, bien qu'il ait été montré dans de nombreux ouvrages savants que ces notions s'analysent sur divers plans : morphologique, lexical, syntaxique, textuel et sémantique (entre autres Bronckart, 1996; Gosselin, 1996; Meleuc \& Fauchart, 1999).

L'objectif de cette contribution est de proposer une démarche d'analyse des productions d'élèves qui puisse outiller l'enseignement des phénomènes grammaticaux complexes liés à l'emploi des temps verbaux dans les textes. La démarche proposée permet de situer ces phé- 
nomènes et d'en donner une explicitation susceptible d'être comprise des élèves. L'outil d'analyse vise à articuler les notions grammaticales et l'activité langagière, en distinguant les différents plans d'analyse du système verbal ${ }^{1}$. L'exposé de notre argumentaire suivra trois étapes. La première partie du texte explicite comment nous abordons la question du fonctionnement des temps du verbe; nous y exposons les présupposés théoriques de la démarche d'analyse des textes d'élèves. La seconde partie présente la démarche d'analyse à l'aide d'exemples concrets tirés de deux corpus : des notes critiques d'une pièce de théâtre écrites par des élèves du secondaire $1\left(9^{\mathrm{e}}\right.$ année $\left.\mathrm{Harmos}^{2}\right)$ et des lettres de demande, rédigées par des étudiants de niveau B2 dans un cours de français langue étrangère. La troisième partie propose une réflexion sur les usages potentiels de cet outil comme dispositif de formation; nous y exposons les avantages et les limites de notre démarche pour le développement professionnel des enseignants, les apprentissages des élèves et le savoir grammatical.

\section{Pourquoi est-il difficile pour les enseignants d'aborder le fonctionnement des temps du verbe dans les textes?}

La non-maitrise du système verbal et des mécanismes de cohésion verbale est à l'origine de plusieurs types d'erreurs dans les copies des élèves et des étudiants universitaires (Meleuc \& Fauchart, 1999). Les lacunes des apprenants découlent de plusieurs facteurs. Un premier ensemble de facteurs relève de la description de la langue. D'abord, les lacunes sont dues à la méconnaissance des distinctions entre les catégories de mode, d'aspect, de temps (Leeman-Bouix, 1994; Tomassone, 2002). Elles résultent également des efforts d'abstraction que requiert la mise en relation de trois époques différentes (présent, passé et futur) et des deux autres repères servant à les définir : le moment de l'énonciation et le moment du procès relaté (Reichenbach, 1947; Moeschler, 1998). Les erreurs proviennent aussi de contraintes grammaticales : on pense

1. Par système verbal, nous entendons l'ensemble organisé des catégories grammaticales liées au verbe (la personne grammaticale, le mode, le temps et l'aspect), représenté par la conjugaison et concernant en particulier les valeurs et l'emploi des modes et des temps verbaux (Chartrand, Aubin, Blain \& Simard, 1999/2011).

2. L'équivalent de la classe de sixième en France. 
par exemple au si hypothétique et à la concordance des temps dans le style indirect au passé (Guénette, Lépine \& Roy, 1995). Certains problèmes ont trait au sens : il peut y avoir une incompatibilité sémantique entre une expression de temps (organisateurs textuels, accélérateurs de rythme, etc.) et le temps du verbe. Un deuxième ensemble de facteurs se rattache à l'enseignement de la grammaire. L'étude traditionnelle du verbe a eu tendance à tout ramener à la chronologie, à la datation du procès, ce qui a contribué à entretenir les confusions entre moment de l'évènement, moment de l'énonciation et moment de référence (Combettes \& Fresson, 1975). Les problèmes de compréhension du système verbal et d'interprétation des mécanismes de cohésion verbale des élèves proviennent également d'une terminologie traditionnelle qui emploie des étiquettes trompeuses pour nommer les différentes formes que peut prendre le verbe (Chartrand, 2011). Cette terminologie contribue à obscurcir les distinctions entre les concepts de mode, d'aspect, de temps et de personne ${ }^{3}$.

\subsection{Genres de textes, types de discours : une sélection d'uenseignables»}

Les problèmes évoqués par Klein (1994) autour de la question du temps dans la langue, à savoir la quantité infinie d'ouvrages sur la question et le fait que la temporalité implique une réflexion à la fois sur le sens, le lexique, les déictiques, le contexte d'énonciation, la morphologie et la syntaxe, exigent de faire des choix au moment d'aborder la question du fonctionnement des temps du verbe dans les textes. Nous avons donc choisi de consacrer notre attention aux questions d'ancrage et d'origine temporelle du discours, car selon nous, celles-ci peuvent être l'objet d'un traitement didactique. En effet, «les modalités concrètes de réalisation des mécanismes de cohésion verbale dépendent fondamentalement des types de discours qu'ils traversent» (Bronckart, 1996, p. 9). Les types de discours entrent dans la composition d'un genre de texte et constituent le produit d'une mise en forme discursive particulière. Aussi, dans le présent texte, nous appelons genres de textes l'ensemble des conditions de production du discours et des attentes typifiantes qui

3. On devrait plutôt faire observer à l'apprenant qu'il y a quatre personnes désignant les locuteurs et interlocuteurs engagés dans la communication (je, tu, nous, vous) et une personne renvoyant à un référent singulier ou pluriel (il/ elle/on - ils/elles). 
caractérisent un ensemble potentiellement illimité d'activités langagières attestées dans une collectivité à une époque donnée, et désignons par types de discours un nombre fini, stable, récurrent et clairement identifiable de modalités de mise en texte qui contribuent à l'organisation des différents segments du texte. Le processus discursif «constitue le créateur de concordance et d'homogénéité; il est doté de sa propre temporalité (axe de référence temporelle) et c'est dans le repérage par rapport à cette temporalité de l'activité discursive que les procès se trouvent eux-mêmes organisés temporellement» (Bronckart, 1996, p. 315).

Les concepts de genre de texte et de type de discours sont donc à la base de notre analyse des productions écrites des élèves quant à la maitrise des phénomènes de cohésion verbale ${ }^{4}$ dans les textes.

\subsection{La démarche d'analyse des textes d'élèves pour situer les capacités et les difficultés}

Notre démarche vise l'analyse de textes d'élèves en vue de dégager les capacités et les difficultés des élèves auteurs de ces textes (Dolz, Gagnon \& Vuillet, 2011). Cette approche donne un autre statut aux erreurs des élèves, en faisant de celles-ci des sources d'informations sur le développement de l'élève. Dans le cas de l'analyse des erreurs en lien avec la cohésion verbale, nous interprétons celles-ci comme des conflits entre les instructions codées par les différents marqueurs de temps dans le texte : verbes, organisateurs textuels, connecteurs, compléments de phrase ou modificateurs du verbe (Gosselin, 1996).

Pour analyser les textes, nous utilisons une grille d'analyse constituée des composantes du genre textuel ainsi que des dimensions liées au fonctionnement de la langue. La modélisation didactique des deux genres étudiés permet une clarification des finalités d'apprentissage relatives à la communication. Les modèles didactiques des genres fournissent une grille des critères et des indicateurs pour évaluer les capacités et les manques des apprenants. Nous analysons les textes des élèves sur le plan de la cohérence au genre textuel, sur le plan des modalités de mise en texte spécifiques, sur le plan du fonctionnement de la langue. Aussi, nous portons un intérêt aux conditions de production de texte

4. Par cohésion verbale, nous entendons, à l'instar de Bronckart, les mécanismes qui «contribuent à l'explicitation des relations de continuité et de discontinuité et/ou d'opposition existant entre les éléments de signification exprimés par les syntagmes verbaux» (1996, p. 277). 
(la consigne de production, la représentation de la situation de communication), nous nous intéressons à la planification du texte, puis aux unités linguistiques en pointant particulièrement la base temporelle choisie, les principaux changements temporels à l'intérieur du texte, les organisateurs textuels et les connecteurs ${ }^{5}$. Concernant les items relatifs au fonctionnement de la langue, notre regard se porte à la fois sur les erreurs d'orthographe, en lien avec les constructions syntaxiques. Pour analyser les questions orthographiques, nous reprenons le classement institué par Catach (1980/1986) et distinguons les erreurs de type phonogrammique, logogrammique et morphogrammique. Pour ce qui est des questions syntaxiques, nous nous intéressons aux choix d'auxiliaires ou de semi-auxiliaires et à la valence syntaxique ${ }^{6}$. Le tableau 1 regroupe les items de la grille d'analyse en vue de l'analyse de textes argumentatifs.

\section{Du fonctionnement des temps verbaux dans deux corpus de textes argumentatifs}

Les textes rassemblés en vue de l'analyse appartiennent à l'activité langagière argumenter, c'est-à-dire qu'ils abordent des problèmes sociaux controversés et visent l'étayage, la réfutation et la négociation de prises de position. Suivant le modèle d'analyse des activités langagières proposé par Bronckart (1996), c'est le type de discours interactif qui y est dominant. Celui-ci se caractérise par un monde référent conjoint au monde ordinaire de l'agent producteur, monde qui n'est ancré à aucune autre origine. Le moment où se déroulent les évènements et le moment où ils sont écrits sont liés. Le discours interactif implique les paramètres de l'acte de production (ou du moins certains d'entre eux). Il y a donc présence de l'énonciateur et du destinataire dans le texte. Aussi, pour l'analyse des mécanismes de cohésion verbale, la durée de l'acte de production peut constituer un paramètre pertinent. Cette pertinence n'exclut pas cependant que soient créés, dans le cours du processus expositif-interactif, des axes de référence temporelle distincts de

5. L'organisateur textuel est une phrase, un groupe ou un mot qui sert le balisage des grandes parties du texte, en marquant la valeur des transitions. Le connecteur est une unité qui intervient au niveau microstructurel du texte (Chartrand, 2008).

6. Par valence syntaxique, nous entendons l'ensemble des propriétés de rection des verbes du point de vue syntaxique. 


\begin{tabular}{|c|c|}
\hline Base d'orientation & Commentaires \\
\hline $\begin{array}{l}\text { - But(s) : Prendre position par rapport à une contro- } \\
\text { verse en justifiant son point de vue par un ensemble } \\
\text { d'arguments. } \\
\text { - Paramètres du contexte de communication : prise en } \\
\text { compte du lieu social de l'énonciation, de l'énoncia- } \\
\text { teur/argumentateur, du destinataire. } \\
\text { - Contenus thématiques. }\end{array}$ & \\
\hline Planification & \\
\hline $\begin{array}{l}\text { - Macro-planification : mise en page, structuration du } \\
\text { texte en parties, ordre des parties du texte. } \\
\text { - Micro-planification : cohérence interne de chacun } \\
\text { des paragraphes : progression thème/rhème (lois de } \\
\text { progression, répétition, non-contradiction). }\end{array}$ & \\
\hline Textualisation & \\
\hline $\begin{array}{l}\text { - Cohésion verbale : base temporelle maintenue; les } \\
\text { autres temps de verbes utilisés sont appropriés. } \\
\text { - Cohésion nominale : phénomènes de reprise. } \\
\text { - Organisateurs textuels et connecteurs. } \\
\text { - Lexique / vocabulaire. } \\
\text { - Marqueurs de modalisation : utilisation du condi- } \\
\text { tionnel, du subjonctif, des auxiliaires de modalité, } \\
\text { des adjectifs et des adverbes. } \\
\text { - Marqueurs de prise en charge énonciative : orchestra- } \\
\text { tion des différentes instances énonciatives : emploi } \\
\text { des déictiques et des pronoms. }\end{array}$ & \\
\hline Fonctionnement de la langue & \\
\hline $\begin{array}{l}\text { - Erreurs phonogrammiques : erreurs dans l'écriture } \\
\text { du lexème verbal (radical) ou de la désinence en lien } \\
\text { à la phonétique. } \\
\text { - Erreurs logogrammiques. } \\
\text { - Erreurs morphogrammiques lexicales (erreurs dans } \\
\text { la graphie du radical) et grammaticales (erreurs } \\
\text { d'accord). } \\
\text { - Erreurs liées à la syntaxe : valence du verbe, choix } \\
\text { de l'auxiliaire ou du semi-auxiliaire. }\end{array}$ & \\
\hline
\end{tabular}

Tableau 1. - Grille d'analyse de textes argumentatifs. 
cette durée de production. Étant donné cette diversité de paramètres de contrôle possibles, l'élaboration de la base temporelle présente, dans le discours interactif, une complexité supérieure à celle des autres types de discours. Le repérage des procès peut s'effectuer par rapport à la temporalité de l'énonciation. D'autres axes de référence peuvent cependant être construits, de façon locale, tout au long du processus expositif-interactif. Dans ces cas de figure, le repérage des procès peut alors s'effectuer soit par rapport à la durée associée à l'acte de production, soit par rapport à cet axe local.

Le premier genre de texte analysé est la note critique d'une sortie au théâtre. Les huit exemplaires du genre ont été produits par des élèves en première année du secondaire ( $9^{\mathrm{e}}$ année Harmos). Le second genre de texte analysé est la lettre de demande. Nous nous intéressons aux lettres rédigées par les étudiants de français langue étrangère du cours Atelier d'écriture à l'École de langue et de civilisation françaises de l'université de Genève. Ces deux genres argumentatifs présentent des caractéristiques génériques qui peuvent être contrastées.

Dans les productions recensées, le repérage des procès s'effectue par rapport à la temporalité de l'acte de production. Les étudiants utilisent donc tous comme temps de base l'indicatif présent. Les procès relatés, celui de la soirée au théâtre pour la note critique et celui de la réception de la décision de la suppression du parcours linguistique $\mathrm{A}$ pour la lettre de demande (cf. 2.2), sont mis en rapport avec d'autres éléments. Et c'est dans cette mise en rapport du procès avec d'autres évènements, de durée variable, antérieurs, simultanés ou postérieurs que se situent les principaux obstacles. Voyons de manière plus précise les productions et les difficultés rencontrées par les apprenants.

\subsection{Les bijoux de la Castafiore : Ia note critique de la sortie au théâtre}

Les textes du corpus Les bijoux de la Castafiore ont la particularité d'appartenir à ce que Combettes et Fresson nomment «des textes hésitants», oscillant entre deux systèmes d'énonciation (1975). En effet, l'énonciateur de la note critique doit d'abord raconter la pièce qu'il a vue et relater ses impressions en vue d'inciter ou non son destinataire à aller voir la pièce. L'objectif d'enseignement de la note critique est donc d'entrainer les élèves à porter un jugement critique sur un produit culturel et à partager leurs avis sur ce produit. Sur le plan de la structure, la note critique comprend une partie informationnelle (les informations sur le spectacle et la bande dessinée), une partie narrative (résumé de la 
pièce) et une partie argumentative (évaluation et appréciation justifiée par des arguments). L'une des principales exigences du travail de ce genre de texte est la prise en compte du destinataire; c'est le destinataire ignorant, à qui il faut fournir suffisamment d'informations et d'arguments, qui conditionne tout le travail d'élaboration de la note critique (Dolz, Noverraz \& Schneuwly, 2001).

La consigne de production à la base des notes critiques analysées est la suivante : les élèves doivent rédiger la note critique de la pièce de théâtre Les bijoux de la Castafiore, inspirée de la bande dessinée du même nom, à laquelle ils ont assisté le vendredi précédent au théâtre de Carouge.

À la lecture des productions réalisées par les élèves du secondaire, on est étonné des différences de qualité entre celles-ci, surtout considérant le fait que les étudiants semblent avoir déjà été en contact avec le genre $^{7}$. Si certaines notes critiques attestent d'une certaine connaissance du genre par les élèves, d'autres, très brèves, semblent avoir été rapidement exécutées. De façon générale, la difficulté principale se situe dans la structuration de la note critique en partie. Les élèves éprouvent des difficultés à lier leur évaluation-appréciation de la pièce et les informations sur celle-ci : l'articulation entre les parties argumentative, informationnelle et narrative du texte est difficile. Ils ont notamment de la peine à situer l'énoncé du commentaire critique et les informations sur la pièce. C'est le cas notamment de la production de Philippe ${ }^{8}$.

(1) «parce que la particularité de cette pièce c'est que l'histoire est quasiment inexistante jusque au moment où la castafiore se fait volé ses bijoux et cette pièce offre la particularité la plus cool est de pouvoir voir une bande dessinée je vous conseille de ne pas aller la voir.»

Si nous restreignons l'analyse à la progression des informations dans ce segment de texte, nous constatons que la proximité, voire l'amalgame,

7. Les informations contenues sur la feuille-support de la consigne nous poussent à penser que les élèves ont déjà rédigé des notes critiques. Le support comprend une série d'indications pour guider l'écriture des élèves ainsi qu'une grille d'autoévaluation portant sur quatre points : 1) la présentation et l'organisation du texte; 2) les informations sur le spectacle et sur la bande dessinée; 3) les arguments critiques et les procédés de persuasion; 4) l'usage de la langue.

8. Tous les prénoms utilisés sont fictifs. Les extraits des productions des élèves sont livrés en l'état, sans correction préalable. 
des informations sur la pièce et de l'avis donne une impression de précipitation au lecteur.

Parfois, c'est la mise en scène de l'énonciation qui pose problème. Marco, un autre élève du groupe, rencontre une difficulté au moment du passage entre le récit du vendredi soir et le commentaire critique :

(2) «Un vendredi soir on est allé au théâtre de carouge. On a vu "Les bijoux de la Castafiore", j'ai bien aimé. Je trouve qu'ils parlaient trop fort. Les arbres étaient trop simple, le reste du décor était bien.»

L'élève a du mal à articuler ces deux segments du texte : le moment de la réception de la pièce marqué par le passé composé «j'ai bien aimé» et celui de l'énonciation «je trouve qu'ils parlaient trop fort». Ces deux moments sont mis dans un rapport de proximité, sans lien explicite, ce qui nuit à la compréhension des propos de l'élève. Les erreurs dans les reprises anaphoriques pronominales témoignent d'ailleurs de ces problèmes de reprise et de progression de l'information. Un peu plus loin dans le texte, la jonction entre le récit des sentiments éprouvés et les commentaires sur le jeu des acteurs se fait aussi difficilement :

(3) «Je trouve que les comédiens jouaient bien. Le personnage que j'ai le plus aimé c'est Bianca, elle joue bien, j'aime bien son rire, elle chante bien.»

L'imparfait dans «les comédiens jouaient bien» sert à décrire les impressions ressenties. Or, l'irruption du présent «elle joue bien, «j'aime», «elle chante» à la suite du passé composé «que j'ai le plus aimé» vient tout d'un coup brouiller les repères temporels construits : la focale se déplace de la description des impressions ressenties par l'énonciateur le soir de la représentation à son avis général sur le travail de la comédienne, sans transition.

Dans certaines copies toutefois, les élèves utilisent les ruptures temporelles afin de créer des effets de sens pour accrocher le lecteur. Dans les textes de Valérie et de Jeanne, le temps du verbe employé dans l'amorce, le futur proche ou le présent, vise à interpeler directement le destinataire :

(4) «Enfin, un vendredi spécial car on va regarder une pièce des Bijoux de la Castafiore au théâtre.

Mais alors, est-ce que, c'était bien? je vous réponds oui c'était génial.» 
(5) «"Tu aime voir des Bande Dessiné mais on(au) théâtre ?” J'ai dit oui pour voir les bijoux de la Castafiore on théâtre parce que je voulais voir si il fesait la même chose que la Bande Dessiné raconte.»

Nous avons noté très peu d'erreurs en lien avec l'orthographe ou la syntaxe. Un élève, Ovide, semble confondre l'écriture des formes verbales «été » et «étaient» :

(6) «Les bijoux de la Castafiore a était joué la première fois en 2001 au théâtre Amstrancrame, et la pièce a était reprise au théâtre de Carouge.»

L'erreur ici nous semble de nature phonogrammique, due à une confusion du son /e/ ou du son / $/ \varepsilon /$. Comme autres erreurs en lien avec le fonctionnement de la langue parmi les exemples recensés, notons des erreurs morphogrammiques lexicales : l'écriture du radical de faire en (5) ; morphogrammiques grammaticales : une confusion entre la forme participe et infinitive du verbe voler en (1); une erreur dans l'écriture de la désinence du verbe aimer à la deuxième personne du singulier en (5).

\subsection{La lettre de demande au directeur du programme}

Les textes analysés proviennent d'une unité de formation intitulée Atelier d'écriture. Il s'agit d'étudiants poursuivant un diplôme d'études de français langue étrangère à la Faculté des lettres de l'université de Genève, à l'École de langue et de civilisation françaises, au cours de l'année universitaire 2011-2012 (désormais ECLF). Parmi les langues d'origine des étudiants, on trouve le russe, le bulgare, l'albanais, le chinois, le japonais, l'anglais, l'allemand et le tagalog. Les objectifs de l'atelier sont les suivants : «assurer les savoirs et savoir-faire du niveau B2 du Cadre européen de référence et exercer ceux du niveau $\mathrm{C} 1 »$. Cela signifie que les étudiants doivent pouvoir écrire des textes clairs et détaillés, de divers genres, en développant un point de vue et en faisant la synthèse d'informations empruntées à des sources diverses. La diversité des textes doit servir l'entrainement de structures linguistiques essentielles.

La consigne de production qui apparait sur la feuille remise aux étudiants se veut réaliste : "Vous aviez l'intention de suivre le parcours A, avec le renforcement linguistique, mais vous venez d'apprendre que la direction de l'ECLF a décidé de le supprimer pour des raisons budgétaires.» Les étudiants doivent donc écrire au professeur Gajo, véritable directeur de l'institution, pour lui demander de revenir sur cette 
décision. La consigne leur demande d'expliquer brièvement leurs motivations et leurs besoins. Les étudiants ont donc rédigé une lettre de demande dans laquelle ils prennent position par rapport à la suppression du parcours A. En accord avec le genre, les productions épousent une mise en page particulière : les textes sont chapeautés par une date, un lieu, le nom du destinataire, ses coordonnées; ils sont signés par leur énonciateur. La présence de formules de salutations et de formules de clôture atteste du respect des conventions du genre. En témoigne aussi le corps des lettres rédigées, qui comprennent généralement une contextualisation de la demande, la demande en elle-même et sa justification.

Du corpus des 16 textes analysés, nous n'avons pas relevé de problèmes récurrents quant à la structure du texte. Les organisateurs textuels se trouvent en nombre limité, sont peu variés, mais leur emploi est pertinent. Comme dans le corpus précédent, les maladresses les plus fréquentes relativement au fonctionnement des temps du verbe dans les textes surviennent au moment des ruptures temporelles. Dans les productions recensées, le repérage des procès s'effectue par rapport à la temporalité de l'acte de production. Les étudiants utilisent donc tous l'indicatif présent comme temps de base. Le procès de «la réception de la décision de la suppression du parcours $\mathrm{A} »$ est mis en rapport avec d'autres éléments. Et c'est dans cette mise en rapport du procès avec d'autres évènements, de durée variable, antérieurs, simultanés ou postérieurs, que se situent les principaux obstacles.

Un premier ensemble d'erreurs relève de la cohésion textuelle. Les erreurs observables en (7) et (8) sont dues au marquage de l'antériorité dans la contextualisation de la demande au directeur.

(7) «Je viens d'apprendre que le parcours A a été annulé. Je le trouve triste surtout pour les étudiants. J'ai choisi ce cours la semaine prochaine.»

La présence du complément «la semaine prochaine» ne permet pas d'identifier l'effet de sens du passé composé : le complément temporel futur entre en conflit avec l'action ponctuelle passée. Ici, on peine à comprendre ce que l'étudiant a voulu dire.

Dans l'exemple (8), l'usage de l'imparfait au passif entraine une rupture avec le contexte d'énonciation et l'absence de lien avec un autre intervalle du contexte nuit à la clarté du propos :

(8) «j'étais tellement déçue de savoir que le parcours A, avec le renforcement linguistique, était supprimé». 
Dans d'autres exemples, le jeu entre les différents temps de verbes utilisés nuit à la stratégie argumentative employée 9 . Dans l'exemple (9), le passé composé «avez changé », bien que syntaxiquement correct, empêche la projection future des effets de la réception de la lettre :

(9) «j'espère que vous avez changé d'avis après avoir lu cette pétition».

Dans l'exemple (10), le passage du conditionnel «devriez réfléchir» au présent «peuvent» et «doit» ne permet pas non plus de comprendre qu'il s'agit de l'énoncé de conséquences futures :

(10) «Vous devriez bien réfléchir cette décision car il y a beaucoup d'étudiants qui ne peuvent plus continuer leurs études ou leurs carrières et la probabilité de trouver un bon travail doit être très difficile.»

Dans l'exemple (11), le passage du subjonctif présent «puisse» au présent «peut» amène une rupture dans l'énoncé de potentialités :

(11) «Je me propose comme bénévole à l'UNIGE à fin de reunir les fonds monétaires qui puisse aider à ouvrir ce cours et qui peut bénéficier à tous les élèves.»

Dans l'exemple (12), le verbe trouver aurait dû être conjugué au futur.

(12) «C'est vrai que ma situation budgétaire est difficile pour instant. Mais je vous jure que je trouve des moyens pour se sortire cette situations.»

L'erreur peut aussi s'interpréter comme un mauvais choix lexical. En effet, l'aspect lexical du verbe trouver marque l'achèvement du procès avec un résultat; un verbe comme chercher qui présente le procès comme dynamique et non résultatif aurait été préférable.

Un second ensemble d'erreurs concerne des aspects propres au fonctionnement de la langue. Les étudiants éprouvent des difficultés à respecter les règles de la concordance des temps dans les phrases complexes. Dans l'exemple (13), c'est la formulation d'un souhait dans les constructions espérer que qui pose problème :

(13) «j'espère que vous pourriez trouver la solution de ce problème budgétaire».

9. Dans les textes des étudiants, l'argumentation est souvent pragmatique : la décision est évaluée en fonction de ses conséquences favorables ou défavorables, autrement dit de son utilité (Bellenger, 1996). 
Les erreurs dans la construction de la phrase complexe surviennent au moment du marquage de l'antériorité entre le moment de la réception de la triste nouvelle et le moment de la nouvelle elle-même :

(14) «Hier soir, j’ai reçu la nouvelle que vous avez décidé de suprimer le cours DE1 à cause des raisons budgétaires.»

(15) «J'ai entendu que le cours du renforcement linguistique est supprimé pour des raisons budgétaires.»

Les verbes des deux subordonnées devraient être, en (14), au plus-queparfait et, en (15), à l'imparfait au passif afin de marquer que les faits sont antérieurs à ceux désignés par les verbes au passé composé «ai reçu» et «ai entendu». Notons que, du point de vue énonciatif, ces deux derniers exemples sont acceptables.

Dans l'exemple (16), c'est l'emploi du «si» hypothétique qui pose problème :

(16) «et je vous serai vraiment reconaissant si vous pourriez faire un petit effort».

Il arrive que les difficultés d'ordre syntaxique s'entremêlent à des difficultés de compatibilité sémantique et de syntaxe. Dans une copie, c'est la valence du verbe croire qui pose problème, mais aussi la combinaison de croire et de sentiments respectueux sans le passage par en l'expression de :

(17) «Dans l'attente de votre décision, que j'espère favorable, je vous prie de croire, Monsieur, mes sentiments respectueux.»

\subsection{Deux corpus contrastés}

L'analyse du contexte de production s'avère cruciale pour comprendre les textes produits et situer les obstacles des élèves. Les deux genres de textes à produire, appartenant tous deux à l'action langagière argumenter, génèrent des difficultés similaires et divergentes.

La note critique demande à l'élève de maitriser certains contenus, certains procédés argumentatifs afin de porter un jugement sur un produit culturel. Peu d'élèves arrivent à les mobiliser, malgré le fait que ce soit là une deuxième production du genre, malgré la présence d'une grille d'autoévaluation. Le réalisme de la situation de communication scolaire de la lettre de demande, sa proximité avec la réalité des étudiants contribuent à leur faire générer facilement des arguments. Si certains confondent quelque peu l'enjeu fictif de celle-ci (on mêle la suppression 
du parcours avec son renvoi personnel, comme dans l'exemple (12)), la plupart se prêtent au jeu avec aisance; en témoignent la gestion des voix énonciatives (la leur et celle des autres apprenants allophones dans la même situation) et la formulation explicite d'une demande.

La lettre de demande au directeur et la note critique de la pièce de théâtre demeurent deux genres scolaires, mais la base d'orientation (à qui j'écris ? et pour parler de quoi ?) de la lettre de demande semble plus facile à se représenter pour les étudiants. Pour la note critique, les élèves ne connaissent pas assez le genre et ne vont pas assez souvent au théâtre pour prendre, par rapport au contenu du texte, la distance nécessaire à son élaboration.

La comparaison des difficultés montre que les deux groupes partagent une même difficulté : la gestion des ruptures temporelles, car, en effet, c'est lors de la mise en rapport du procès qui fait l'objet d'un commentaire avec d'autres évènements de durée variable, antérieurs, simultanés ou postérieurs, que se situent les principaux obstacles. Les nombreux axes temporels témoignent de la complexité de l'élaboration de la base temporelle présente du discours interactif dans les deux corpus.

D'autres erreurs sont propres au genre travaillé : l'articulation des différents segments du texte dans la note critique et l'expression des formules de souhait ou de salutations finales dans la lettre de demande.

Les erreurs dans la construction de phrases complexes semblent être plus fréquentes chez les étudiants de français langue étrangère, mais cela est dû au fait que les élèves du secondaire emploient généralement des phrases simples et lient les phrases entre elles par juxtaposition, coordination plutôt que par subordination.

\section{Des usages potentiels de notre démarche d'analyse dans la formation des enseignants du secondaire}

En quoi ce travail d'analyse pourrait-il servir à la formation des enseignants? Nous voyons deux grands apports : primo, la démarche permet de sélectionner les concepts et notions nécessaires au travail sur le fonctionnement des temps du verbe dans les textes, clarifiant la métalangue; secundo, en croisant grammaire et production textuelle, elle incite les enseignants à adopter une double perspective dans leur pratique : la prise en compte des besoins des élèves ou des étudiants et le déploiement de l'objet à enseigner. 


\subsection{Clarifier la métalangue}

Un des objectifs d'une formation des enseignants pour traiter un phénomène complexe comme le fonctionnement des temps du verbe est de rendre compte du discours servant à nommer et à présenter les composants de l'objet système verbal. L'analyse dirigée de ces deux corpus permet d'effectuer le tri des concepts et des notions en fonction des nécessités. Les concepts de types de discours et de genres textuels permettent de mettre en évidence, en situation de formation, les paramètres du contexte et de la situation de communication (l'énonciateur, le destinataire et le but), l'organisation du texte et les unités linguistiques. Or, si le genre textuel est un concept fréquent dans les plans d'études ou les manuels, celui de type de discours semble appartenir à une littérature plus savante. Il faudrait alors simplifier et, à l'instar de Combettes, Fresson \& Tomassone (1978) dans leur manuel, reprendre la distinction de Benveniste entre discours et récit, et recourir à des critères simples tels que la correspondance auteur-émetteur, l'interpellation du destinataire, les temps utilisés. Les exemples relevés dans l'analyse montrent la difficulté à mettre en lien les différents temps du texte : le moment où l'on argumente (énonciation), le procès relaté (l'évènement principal, mais aussi les autres procès qui s'y adjoignent) et le moment de référence (le moment dont je parle). En effet, c'est au moment où survient une rupture par rapport au temps de base que les difficultés apparaissent. Il peut être intéressant de faire travailler les apprenants, à partir de leurs propres écrits ou de ceux de leurs pairs, à la mise en relation de ces trois moments, de durée variable. On les amène ainsi à distinguer expression du temps et temps verbaux. En amont, on peut prolonger la réflexion en leur faisant identifier le temps absolu, c'est-à-dire la structuration du temps opérée par le calendrier, le temps anaphorique, lié au moment de référence, et le temps déictique, propre à l'énonciation (Barcelo \& Bres, 2006).

L'analyse a aussi permis de mettre en évidence des indicateurs propres au texte et d'autres relatifs au fonctionnement de la langue. La séparation entre ce qui relève d'une grammaire de la phrase ou de ce que nous avons appelé le fonctionnement de la langue et d'une grammaire pour l'étude du texte demeure assez complexe, même pour des étudiants en formation à l'enseignement. Or, dans l'étude des phénomènes de cohésion temporelle, ces deux plans d'analyse doivent être distingués. Par exemple, le formé doit pouvoir différencier l'organisateur textuel du connecteur. De même, l'apprenant doit reconnaitre les 
situations où s'appliquent les règles de la concordance des temps, de manière à ne pas confondre les notions de cohérence temporelle et de concordance des temps.

\subsection{Aider l'ingénierie didactique en décryptant les contextes communicatifs dans lesquels on fait rédiger les élèves}

Un dispositif de formation basé sur une démarche d'analyse de textes d'élèves incite à la prise en compte des capacités présentes et des besoins des élèves ou des étudiants. Il permet une première identification de problèmes récurrents dans l'apprentissage des phénomènes de cohésion temporelle et de situer ceux-ci dans l'ensemble des composantes de l'objet à enseigner. Il vise à faire entrer l'apprenant en formation dans des cadres d'explicitation multiples : celui du déploiement de l'objet de savoir, celui de l'apprentissage des élèves, en fonction de leur âge, de leur langue première, de leurs capacités. La création d'un outil pour guider les enseignants formés à la lecture et l'analyse des textes fait valoir l'idée qu'un des objectifs de l'enseignement grammatical est d'aider les élèves à produire et à comprendre divers genres de textes. L'usage de textes d'élèves en formation contribue à articuler davantage le terrain au savoir grammatical. Enfin, comme il s'agit de montrer l'usage des notions en contexte, nous contribuons à doter les formés d'une certaine lucidité didactique ${ }^{10}$ devant ce phénomène grammatical complexe.

Comme la démarche d'analyse permet la mise à distance de situations d'écriture, dans des contextes différents, elle expose le contexte communicatif dans lequel on fait écrire les élèves et le met en débat. Du coup, les enseignants en formation sont amenés à revoir certains gestes : la formulation de consignes, la conception de supports (moyens matériels) pour faire écrire, la fictionnalisation de la situation de communication (le comment on va faire comme si), la prise en compte des capacités initiales des apprenants. Qui plus est, le contraste de productions d'élèves francophones et allophones apporte quelques pistes en vue d'un enseignement grammatical tenant compte du contexte francophone plurilingue suisse.

Dans le contexte de la formation, une telle démarche permet d'élaborer un cadre de réflexion sur le genre produit par l'élève, mais aussi sur le dispositif d'enseignement (supports matériels et discours) qui a

10. Nous empruntons l'expression à Jean-Louis Chiss. 
mené à la production du texte. La distinction de ces différentes composantes permet de mieux découper les objets enseignables et de penser les liens entre, d'une part, les différents concepts que l'enseignant doit maitriser pour enseigner et, d'autre part, les notions qu'il doit enseigner ${ }^{11}$ aux élèves. Enfin, la démarche facilite le relai des outils et des contenus de la formation à la classe de deux manières différentes. Comme elle prend appui sur des activités menées en classe, nous supposons qu'elle génèrera de l'intérêt au moment de sa réception en formation. Mais, surtout, cet appui sur le terrain donne de la résonance à la démarche et aux contenus présentés dans les pratiques existantes des enseignants (Gagnon, 2010).

\subsection{Tester la démarche dans le contexte de la formation}

$\mathrm{La}$ «reconnaissance pleine et entière de la fonction métalinguistique et la construction en classe des objets grammaticaux [...] fondée sur la description et l'explicitation des fonctionnements (Chiss, 2005, p. 84) dépendent en grande partie du traitement de ces objets dans la formation des enseignants. Il s'agit de penser des ponts entre formation et enseignement pour qu'un concept grammatical complexe tel que le système verbal devienne objet de formation et puisse être ensuite reformulé dans les pratiques enseignantes. Ce concept complexe peut permettre de mener en formation une «vraie réflexion sur l'enseignable et les degrés d'enseignabilité» (Chiss, 2011, p. 26). L'étude d'un phénomène grammatical complexe, tel que le fonctionnement des temps verbaux dans les textes, requiert donc la mise en place de dispositifs de formation axés sur une démarche active de découverte où les futurs enseignants observent, manipulent, formulent des hypothèses pour développer des concepts et notions (Chartrand, 1996). C'est d'ailleurs à l'expérimentation concrète de notre dispositif sur le terrain que nous consacrerons les prochaines étapes de ce projet.

11. Nous distinguons donc les concepts, produits par le monde scientifique et savant, des notions, lesquelles correspondent à des connaissances générales de sens commun ou d'expérience construites, entre autres, dans une discipline scolaire en classe (Chartrand \& De Koninck, 2009). 


\section{RÉFÉRENCES BIBLIOGRAPHIQUES}

Barcelo, J. \& Bres, J. (2006). Les temps de l'indicatif. Paris : Orphys.

BEACCO, J.-C. (2010) : La didactique de la grammaire dans l'enseignement du français et des langues. Paris : Didier, coll. «Langues et didactique».

Bellenger, L. (1996). L'argumentation. Des techniques pour convaincre. Paris : ESF.

Bronckart, J.-P. (1996). Activité langagière, textes et discours. Pour un interactionisme socio-discursif. Lausanne-Paris : Delachaux et Niestlé.

CATACH, N. (avec C. Gruaz \& D. Duprez). (1980/1986). L'orthographe française : traité théorique et pratique. Paris : Nathan.

Chartrand, S.-G. (dir.). (1996). Pour un nouvel enseignement de la grammaire. Montréal : Les Éditions Logiques.

Chartrand, S.-G. (2008). Progression dans l'enseignement du français langue première au secondaire québécois. Répartition des genres textuels, des notions, des stratégies et des procédures à enseigner au secondaire. Québec Français, numéro hors série.

Chartrand, S.-G. (2011). L'enseignement de la conjugaison : de fausses évidences. Correspondances, 16 (2), 6.

Chartrand, S.-G., Aubin, D., Blain, R. \& Simard, Cl. (1999/2011). Grammaire pédagogique du français d'aujourd'hui. Montréal : Chenelière Éducation.

Chartrand, S.-G. \& De Koninck, G. (2009). La clarté terminologique pour plus de clarté et de rigueur dans l'enseignement du français (suite). Québec Français, 154, 143-145.

Chartrand, S.-G. \& De Pietro, J.-F. (2012). Vers une harmonisation des terminologies grammaticales scolaires de la francophonie : quels critères pour quelles finalités? Enjeux, 84, 5-31.

Chiss, J.-L. (2005). Sciences du langage : le retour. Dans J.-L. Chiss, J. David \& Y. Reuter (dir.), Didactique du français : Fondements d'une discipline (p. 82-94). Bruxelles : De Boeck.

Chiss, J.-L. (2011). La «crise du français» dans la «crise» de l'école : peuton repenser le débat? Dans J.-L. Chiss, H. Merlin-Kajman \& C. Puech (dir.), Le français, discipline d'enseignement : histoire, champ et terrain (p. 17-30). Paris : Riveneuve Éditions.

Combettes, B. \& Fresson, J. (1975). Quelques éléments pour une linguistique textuelle. Pratiques, 6, 25-55.

Combettes, B., Fresson, J. \& Tomassone, R. (1978). Bâtir une grammaire (classe de $5^{e}$ et $\left.6^{e}\right)$. Paris : Delagrave, coll. «G. Belloc».

Dolz, J., Noverraz, M. \& Schneuwly, B. (2001). Séquences didactiques pour l'oral et pour l'écrit. Notes méthodologiques (Vol. IV, 7e/8 $/ 9^{\mathrm{e}}$ ). Bruxelles : De Boeck. 
Dolz, J., Gagnon, R. \& Vuillet, Y. (2011). Production écrite et difficultés d'apprentissages. Genève : Université de Genève, coll. «Carnet des Sciences de l'éducation».

GaGnOn, R. (2010). Former à enseigner l'argumentation orale : de l'objet de formation à l'objet enseigné en classe de culture générale (Thèse de doctorat). Université de Genève.

Gosselin, L. (1996). Sémantique de la temporalité en français. Un modèle calculatoire et cognitif du temps et de l'aspect. Paris : Duculot, coll. «Champs linguistiques».

Guénette, L., LéPIne, F. \& Roy, R. L. (1995). Le français tout compris : guide d'autocorrection du français écrit. Montréal : ERPI.

KLEIN, W. (1994). Time in language. Londres : Routledge.

Leeman-Bouix, D. (1994). Grammaire du verbe français. Des formes au sens. Paris : Nathan Université.

Marmy Cusin, V. (2012). Développer et comprendre des pratiques d'enseignement de la grammaire intégrées à la production textuelle : entre les dires et les faires (Thèse de doctorat en sciences de l'éducation). Université de Genève.

Meleuc, S. \& Fauchart, N. (1999) : Didactique de la conjugaison. Le verbe «autrement». Lonrai : Bertrand-Lacoste/CRDP Midi-Pyrénées.

Moeschler, J., Jayez, J., Kozlowska, M., Luscher, J.-M., de Saussure, L. \& Sthioul, B. (1998). Le temps des événements. Pragmatique de la référence temporelle. Paris : Kimé.

Reichenbach, H. (1947). Symbolic logic. Berkeley : University of California Press.

Schneuwly, B. \& Dolz, J. (avec S. Aeby-Daghé, D. Bain, S. CanelasTrevisi, G. Sales Cordeiro, R. Gagnon, M. Jacquin, C. Ronveaux, T. Thévenaz-Christen \& S. Toulou). (2009). Des objets enseignés en classe de français. Le travail de l'enseignant sur la rédaction de textes argumentatifs et sur la subordonnée relative. Rouen : Presses universitaires de Rouen.

Tomassone, R. (2002). Pour enseigner la grammaire. Paris : Delagrave Édition. 